\title{
Erythrocyte rouleau formation under polarized electromagnetic fields
}

\author{
José Luis Sebastián, Sagrario Muñoz San Martín, Miguel Sancho, and José Miguel Miranda \\ Departamento de Física Aplicada III, Facultad de Ciencias Físicas, Universidad Complutense, 28040 Madrid, Spain \\ Gabriel Álvarez \\ Departamento de Física Teórica II, Facultad de Ciencias Físicas, Universidad Complutense, 28040 Madrid, Spain
}

(Received 31 March 2005; published 21 September 2005)

\begin{abstract}
We study the influence of an external electromagnetic field of $1.8 \mathrm{GHz}$ in the formation or disaggregation of long rouleau of identical erythrocyte cells. In particular we calculate the variation of the transmembrane potential of an individual erythrocyte illuminated by the external field due to the presence of the neighboring erythrocytes in the rouleau, and compare the total electric energy of isolated cells with the total electric energy of the rouleau. We show that the polarization of the external electromagnetic field plays a fundamental role in the total energy variation of the cell system, and consequently in the formation or disaggregation of rouleau.
\end{abstract}

DOI: 10.1103/PhysRevE.72.031913

PACS number(s): 87.18.Ed, 87.50.-a, 02.70.Dh

\section{INTRODUCTION}

It is well known that erythrocytes in low shear flow can aggregate and form a close-packed stack of cells, the socalled rouleau. These red cells that appear stacked like coins can be easily observed using a dark field microscope. (Rouleau must not be confused with erythrocyte aggregation, also known as blood sludge; this last condition is one step worse than rouleau and it is often seen in patients with degenerative diseases.) The length of an erythrocyte rouleau (up to $50 \mu \mathrm{m})$ is determined by the repulsive force between the negatively charged cells, by the cell-to-cell adhesion of their flat surfaces induced by plasma proteins and by the disaggregating shear force generated by blood flow [1]. Under normal circumstances the blood flow is sufficient to disperse erythrocyte rouleau, and this process is essential for normal tissue perfusion. However, in low-flow states and other pathological conditions rouleau of erythrocytes may contribute to circulatory disorders, in particular microcirculation, leading to occlusion of microvessels. Rouleau can also drastically hinder oxygen transportation, resulting in a decrease of circulation which may cause chronic fatigue, poor digestion, and edema. The importance of these rouleau effects explains why they attract considerable research interest.

Skalak et al. [2] have studied the formation of rouleau with flat contact surfaces between the erythrocytes, and therefore limited to cell shape geometries with equatorial mirror symmetry. More recently and based on the computational approach that Bozic et al. [3] developed for the study of lipid membranes, Derganc et al. [4] have extended the work of Skalak et al. to include all possible axisymmetric equilibrium shapes of erythrocytes deformed from their normal biconcave shape, including mirror-symmetric discocytes and cup-shaped stomatocytes in a long rouleau of identical cells.

If situations such as high-shear flow or echinocyte transformation are not considered, the deformations of erythrocytes in the rouleau formation are very small and the skeleton elasticity can be safely omitted from the analysis [5]. Therefore, the equilibrium state of erythrocytes in the rouleau corresponds to the minimum of the sum of the adhesion energy, the elastic energy of their membranes [1] and the basic electric energy (due to the resting transmembrane potential). From the previous statement it can be inferred that rouleau formation will be favored if the total energy of an erythrocyte cell system is decreased. This energy variation may originate from different sources both internal and external to the cell system. In particular, an electromagnetic (EM) field impinging on a biological structure may constitute a main external source.

Exposure of a biological cell to EM fields can produce a variety of profound biochemical and biophysical responses. Weak electric field effects have generally been attributed to field interaction with either membrane or glycocalix constituents. Ongoing research about possible mechanisms of interaction of EM fields with biological tissues and cells in culture has motivated a growing need for accurate models describing the electric behavior of cells exposed to these fields. The magnitude of transmembrane potential (TMP) (the voltage "drop" or the difference in voltage between one face of a bilayer and its immediate opposite face) and the deposited energy are basic issues to understand the relation between the exposition to fields and the subsequent physiological reactions at the cell level $[6,7]$. The transmembrane potential plays a very important role in all cell activity as it controls the transmembrane flow of charged solutes and the activity of voltage-gated ion channels. A variation of the transmembrane potential leads to a local distortion of the field in the cell and its vicinity.

In previous works $[8,9]$ we have already shown that the membrane is a site of high field amplification and that the detailed geometry and electrical properties of the cell can affect the accuracy of the predictions of the electric behavior. In particular, we have shown that the low membrane conductivity (several orders of magnitude lower than those of the cytoplasm and the physiological extracellular medium) implies that most of the electric field and energy within the cell are concentrated on the membrane. Therefore, the knowledge of the electric field distribution within the cell membrane or the equivalent transmembrane potential is of primary importance. This is especially so for erythrocyte rouleau under radio frequency exposure, as the total energy of the cell sys- 
tem is varied and the internal electric field can play an important role in the formation or disaggregation of the rouleau.

Note that in general attractive forces between electrically polarized particles lead to the formation of chainlike aggregates termed "pearl chains" by Schwan and co-workers [10-13], who proposed a theory based on dipole interactions among spherical particles. This theory shows that the formation of pearl chains is characterized by a time constant proportional to the square of the field strength and by a threshold field which depends on the size and elastic properties of the particles and the medium $[10,12]$. Since the induced dipole moment on a cell depends both on the volume and on the square of the field strength, the threshold field to overcome thermal agitation is proportional to $R^{-3 / 2}$, where $R$ stands for the radius of the particles. This dependence has been observed in spherical particles [14] as well as in bacteria and in erythrocytes [15] (in these cases, $R$ is the geometric mean of the major and minor semiaxes). To induce aggregation of erythrocytes Schwan [16] gives a threshold of about $1000 \mathrm{~V} / \mathrm{m}$ at $1 \mathrm{MHz}$. We are not aware of published values for the threshold field at $1.8 \mathrm{GHz}$ but it is likely to be higher. Takashima and Schwan [13] confirm that threshold values for $10 \mu \mathrm{m}$ cells are about $1000 \mathrm{~V} / \mathrm{m}$, but for $10 \mathrm{~nm}$ macromolecules the fields rise up to $1000 \mathrm{kV} / \mathrm{m}$, of the same order of the fields required for complete orientation due to the existence of a typical dipole moment of 10-100 D.

A different approach was taken by Sauer [14,17], who evaluated the Maxwell stress tensor for two approaching spherical particles, and by Foster and Sowers [18] who introduced the membrane into the model and studied the electrically induced force on two shelled spheres and the related hydrodynamic problem of the rate of approach of the cells using a numerical boundary element method.

The aim of the present paper is to perform a study of the influence of an external EM field in a long rouleau of identical erythrocyte cells, and especially to quantify the effects pertaining to a realistic model with a realistic shape of the erythrocyte that cannot be accounted for by a simple dipoledipole interaction. We have selected an operating frequency of $1.8 \mathrm{GHz}$ and a normalized value of the electric field of $1 \mathrm{~V} / \mathrm{m}$ as good representatives of typical values in recent studies of biological effects and industrial applications in the high-frequency range [19-22]. Our numerical calculations can be extended readily for higher field strength values.

The layout of the paper is as follows. In Sec. II we briefly discuss a realistic geometrical model for an erythrocyte cell parametrized in terms of Jacobi elliptic functions. Section III contains a detailed description of the finite element with adaptive mesh numerical technique that has been used to calculate the induced fields within the erythrocytes exposed to a linearly polarized EM plane wave. In Sec. IV we analyze the effects of erythrocyte mutual interactions in rouleau formation. In particular we illustrate how the transmembrane potential of an individual erythrocyte illuminated by the external EM field is modified by the close presence of neighboring erythrocytes, and compare the total electric energy of isolated cells with the total electric energy of the rouleau. Although we will summarize our conclusions in the final Sec. V, we anticipate here that the polarization of the external
EM field plays a fundamental role in the total energy variation of the cell system because field-induced aggregation is favored along the field lines.

\section{ERYTHROCYTE MODEL}

The interaction of an external electromagnetic field with biological cells has been the subject of numerous investigations, usually with simple cylindrical or spheroidal geometries to model the cells. The revolution symmetry of these geometries greatly simplifies the calculation of the different quantities of interest, such as electric fields, transmembrane potentials or polarizabilities. However, in order to gain a good insight into the mechanisms of action of electromagnetic fields (including athermal effects) more realistic models must be used. In fact, we have previously shown the crucial role played by the geometry of the cell model in the determination of the electric field, and we have also pointed out that a uniformly shelled ellipsoid is a very crude approximation if we require a precise simulation of the bioeffects in the transition from a normal to an ellipsoidal erythrocyte [23]. More precise shapes, in turn, require highly refined calculation techniques with considerably higher computing times.

The human erythrocyte normal resting shape is a flattened biconcave disk of approximately $8 \mu \mathrm{m}$ in diameter. This particular shape provides the erythrocyte with the necessary flexibility and deformability to traverse the microvasculature. There are several methods to model the complicated erythrocyte shape and deformations thereof. The surface of revolution generated by a Cassini curve [24] is a good and frequently used approximation to the real shape of the erythrocyte cell [23,25]. Its main advantage is that the Cassini curves have simple implicit (as a fourth degree polynomial) and parametric (in terms of trigonometric functions) representations; its main limitation is that a Cassini curve is determined by two parameters which can be used to fit, say, only the length and height of a real erythrocyte.

At the other end in complexity is the approach proposed by Bloor and Wilson [26], who use an elliptic partial differential equation in combination with a model for the surface energy to produce a most realistic representation of the shape of the membrane surface (incidentally, in this method shape parameters are introduced through the boundary conditions).

However, we do not find convenient to forsake the advantages of a simple parametric representation, in particular considering that any further shape transformations are readily amenable (for example, those used to model changes in shape membranes which occur spontaneously when cells are immersed in an aqueous environment under appropriate conditions [27]). For this reason, in the present study we have opted for a simple generalization of the model proposed by Kuchel and Fackerell [28], whereby the shape of a biconcave erythrocyte is represented by a set of parametric equations in terms of the $\operatorname{sn}(u, m), \operatorname{cn}(u, m)$, and $\operatorname{dn}(u, m)$ Jacobi elliptic functions [29] with three free parameters.

Our model of the erythrocyte cell is formed by two media: the membrane and the cytoplasm. The cytoplasm is a physiological saline solution with a protein volume fraction of 0.26 . The membrane will be represented by a shell of con- 
TABLE I. Relative permittivity $\epsilon / \epsilon_{0}$, conductivity $\sigma$, and loss tangent for the cytoplasm, membrane and external continuous medium at $1.8 \mathrm{GHz}$ (see Ref. [35]).

\begin{tabular}{cccc}
\hline \hline Medium & $\epsilon / \epsilon_{0}$ & $\sigma(\mathrm{S} / \mathrm{m})$ & Loss tangent \\
\hline Cytoplasm & 50 & 0.53 & 0.10590 \\
Membrane & 9.04 & $1 \times 10^{-6}$ & $1.1052 \times 10^{-6}$ \\
External medium & 80 & 0.12 & 0.014986 \\
\hline \hline
\end{tabular}

stant thickness $\delta=8 \mathrm{~nm}$ that has a very low conductivity and a frequency-independent relative permittivity [30,31]. The cell is immersed in an external continuous medium (the radiation region) formed by an electrolyte with the dielectric properties of physiological saline. Table I shows the electrical parameters for the membrane, cytoplasm and external medium. These values are typical for the erythrocyte structure and have been extensively used in the literature [30-35].

The cytoplasm is bounded by the surface

$$
\begin{aligned}
& \mathbf{r}(u, \phi) \\
& \quad=(a \operatorname{cn}(u, m) \cos \phi, a \operatorname{cn}(u, m) \sin \phi, \pm b \operatorname{sn}(u, m) \operatorname{dn}(u, m)),
\end{aligned}
$$

$$
u \in[0, U], \quad \phi \in[0,2 \pi],
$$

where the plus and minus signs correspond to the upper and lower half of the cell, respectively, and where the three free parameters are $a, b$ and $m$. By putting $(u, \phi)=(0,0)$ into Eq. (1) we find that $a=l / 2$, where $l$ is the diameter of the erythrocyte. The range $[0, U]$ of the parameter $u$ is determined by the condition $\operatorname{cn}(U, m)=0$, which in turn yields $b$ $=h_{0} /[\operatorname{sn}(U, m) \operatorname{dn}(U, m)]$, where $2 h_{0}$ is the height of the erythrocyte at its center. Finally, $m$ can be fixed by the condition $h_{\max }=b \operatorname{sn}\left(u_{\max }, m\right) \operatorname{dn}\left(u_{\max }, m\right)$, where $2 h_{\max }$ is the maximum height of the erythrocyte (reached at the value $u_{\max }$ of the variable $u$ ). General expressions of these parameters in terms of elliptic integrals can be found in Ref. [28]. The dimensions of the biconcave erythrocyte used in this work are $l=7.8 \mu \mathrm{m}, h_{0}=0.5 \mu \mathrm{m}$, and $h_{\max }=0.85 \mu \mathrm{m}$, which correspond to $a=3.9, b=1.61669$, and $m=0.90435$. Finally, we generated the uniform shell representing the membrane by shifting the cytoplasm surface a constant distance $\delta$ along the normal to the surface at each point

$$
\mathbf{r}_{\delta}(u, \phi)=\mathbf{r}(u, \phi)+\delta \mathbf{n}(u, \phi),
$$

where $\mathbf{n}(u, \phi)$ is the outward unit normal to the surface given by

$$
\mathbf{n}(u, \phi)=-\frac{\frac{\partial \mathbf{r}}{\partial u} \times \frac{\partial \mathbf{r}}{\partial \phi}}{\left\|\frac{\partial \mathbf{r}}{\partial u} \times \frac{\partial \mathbf{r}}{\partial \phi}\right\|} .
$$

The minus sign in Eq. (3) is necessary because as the variable $u$ increases from 0 to $U$, both $x(u, \phi)$ and $y(u, \phi)$ decrease from $l / 2$ to 0 . The three-dimensional (3D) erythrocyte cell model obtained by this method, with the corresponding dimensions and (for future reference) incident EM plane wave polarizations can be seen in Fig. 1 .

\section{CALCULATION OF THE INTERNAL ELECTRIC FIELD DISTRIBUTION AND TRANSMEMBRANE POTENTIAL}

In low shear flow conditions, the total energy of the erythrocytes in the rouleau can be approximated as the sum of three independent contributions: the membrane elastic energy, the adhesion energy and the cell basic electric energy. Furthermore, in the aforementioned conditions the deformations of the erythrocytes (whose mechanical properties arise from the elastic properties of its lipid bilayer and the underlying membrane skeleton) in the rouleau formation are very small and can be safely omitted from the analysis.

The first contribution to the total energy has been studied in detail by several authors (Bozic et al. [3], Miao et al. [36]), who used the area difference elasticity (ADE) model. Similarly, Derganc et al. [4] and Seifert et al. [37] have analyzed the strength of adhesion between neighboring cells and vesicles, respectively. In the present work we focus on the variations of the total energy due to the third contribution, i.e., to variations of the rouleau electric energy from the cell resting transmembrane potential when the cells are exposed to a linearly polarized external electromagnetic field. Implicit in our subsequent calculations is the further reasonable as-

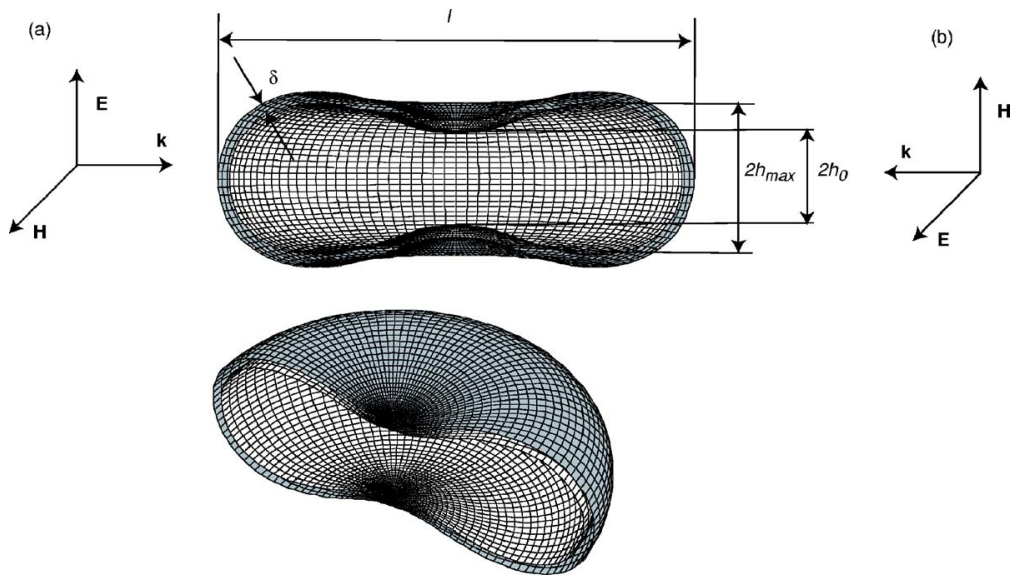

FIG. 1. (Color online) Dimensions and 3D geometrical model used to shape the biconcave erythrocyte $\left(\delta=8 \mathrm{~nm}, l=7.8 \mu \mathrm{m}, h_{0}=0.5 \mu \mathrm{m}\right.$, $\left.h_{\max }=0.85 \mu \mathrm{m}\right)$. The polarization of the incident EM plane wave is (a) electric and (b) magnetic. 

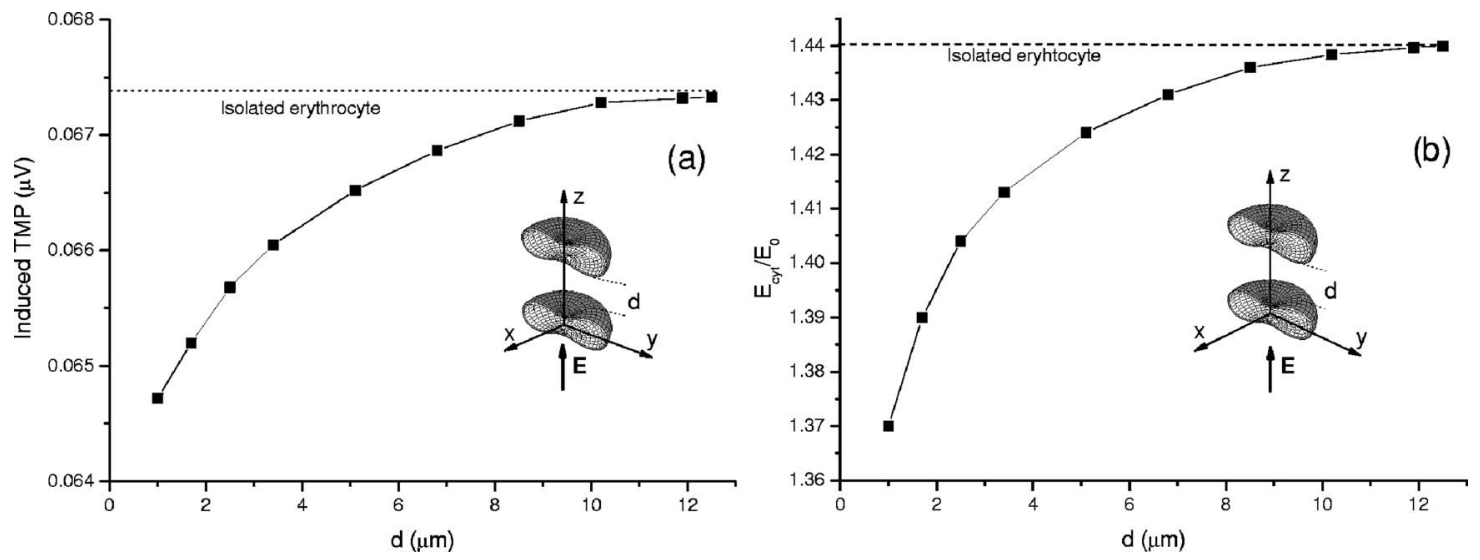

FIG. 2. Mutual interaction between two erythrocytes. Variations of (a) the induced transmembrane potential at $x=0$ and (b) the field within the cytoplasm as a function of the separation $d$ at $x=y=0$. The external field of $1 \mathrm{~V} / \mathrm{m}$ is parallel to the minor axis and the cells are stacked along this direction, as is typical in rouleau formation.

sumption that under the exposure to the external EM field both the elastic and the adhesion energy contributions to the rouleau energy remain unaltered $[4,38]$. Therefore, any variation in the total rouleau energy can be attributed to the interaction of the rouleau with this external EM field.

The cell basic electric energy is modified by the presence of adjacent cells and by the fields induced in the membrane and in the cytoplasm by the external field. We can obtain an analytic estimate of this energy by a somewhat crude model in which the erythrocyte is considered as a spherical shell of thickness $\delta$ whose radius $R$ is chosen so that the volume of the spherical erythrocyte be equal to the volume of the real erythrocyte. The (complex) dipolar moment $\mathbf{p}$ induced in this shelled sphere by an external field $\mathbf{E}$ is [39]

$$
\mathbf{p}=4 \pi R^{3} \hat{\epsilon}_{\mathrm{ext}} \frac{\hat{\epsilon}_{\mathrm{eff}}-\hat{\epsilon}_{\mathrm{ext}}}{\hat{\epsilon}_{\mathrm{eff}}+2 \hat{\epsilon}_{\mathrm{ext}}} \mathbf{E}
$$

where

$$
\hat{\epsilon}_{\mathrm{eff}}=\hat{\epsilon}_{\mathrm{mem}} \frac{\left(\frac{R}{R-\delta}\right)^{3}+2 \frac{\hat{\epsilon}_{\mathrm{cyt}}-\hat{\epsilon}_{\mathrm{mem}}}{\hat{\epsilon}_{\mathrm{cyt}}+2 \hat{\epsilon}_{\mathrm{mem}}}}{\left(\frac{R}{R-\delta}\right)^{3}-\frac{\hat{\epsilon}_{\mathrm{cyt}}-\hat{\epsilon}_{\mathrm{mem}}}{\hat{\epsilon}_{\mathrm{cyt}}+2 \hat{\epsilon}_{\mathrm{mem}}}},
$$

and where $\hat{\epsilon}_{\mathrm{ext}}, \hat{\epsilon}_{\mathrm{mem}}$, and $\hat{\epsilon}_{\mathrm{cyt}}$ are the complex permittivities $\hat{\epsilon}=\epsilon-i \sigma / \omega$ of the external medium, of the membrane and of the cytoplasm, respectively. The interaction energy between two such spheres is

$$
W=-\frac{1}{4 \pi \epsilon_{\mathrm{ext}}} \frac{\operatorname{Re}\left(\mathbf{p}_{1} \mathbf{p}_{2}^{*}\right)}{r^{3}}
$$

where "Re" stands for the real part. For $R=2.53 \mu \mathrm{m}$ (i.e., an sphere of $68 \mu \mathrm{m}^{3}$, the volume of our realistic model), tangential spheres (i.e., $r=2 R$ ) and an electric field of $1 \mathrm{~V} / \mathrm{m}$, Eq. (6) yields an interaction energy $W=-4.0 \times 10^{-28} \mathrm{~J}$. However, if we disregard the interpenetration of the spheres and place the induced dipoles at a distance equal to the distance between the centers of the erythrocytes in the rouleau $r=2 h_{\max }=1.7 \mu \mathrm{m}$ the interaction energy is $W=-1.0$
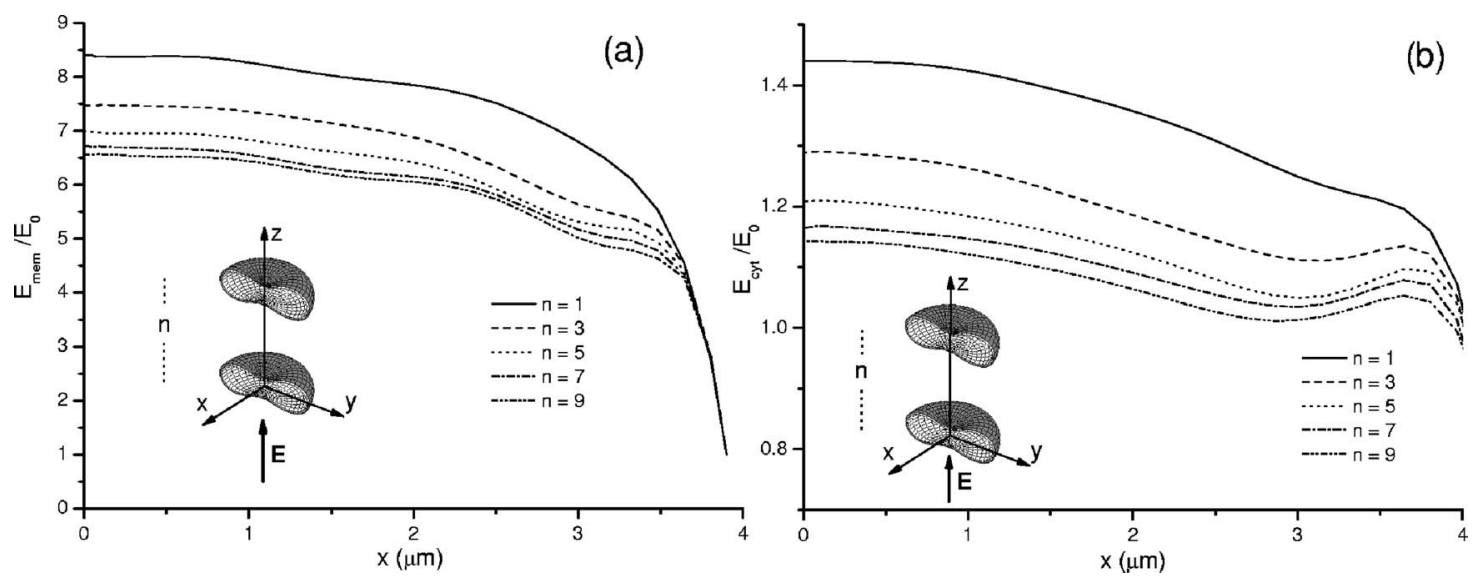

FIG. 3. Variation of the electric field intensity along the major axis within (a) the membrane and (b) the cytoplasm of the central cell of the rouleau (not shown in the insets) for different numbers $n$ of cells of the rouleau. The external field is "electrically" polarized (i.e., parallel to the minor axes of the cells). 

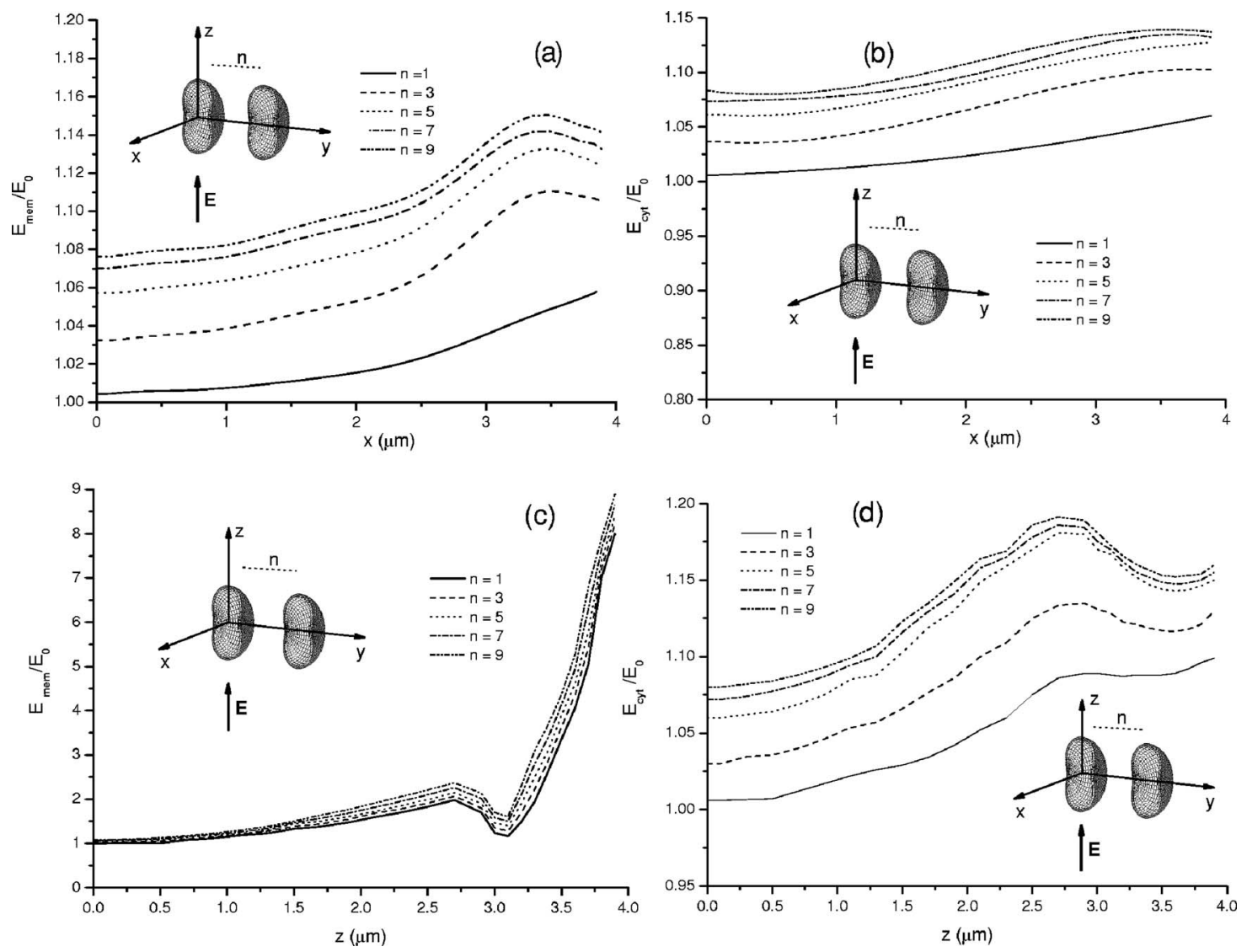

FIG. 4. Variation of the electric field intensity in the membrane and in the cytoplasm along two perpendicular major axes of the central cell of the rouleau (not shown in the insets) for different numbers $n$ of cells in the rouleau. The external field is "magnetically" polarized. (a) Electric field in the membrane as a function of the coordinate labeled $x$ for $z=0$ in the accompanying inset; (b) electric field in the cytoplasm as a function of the coordinate labeled $x$ for $z=0$ in the accompanying inset; (c) electric field in the membrane as a function of the coordinate labeled $z$ for $x=0$ in the accompanying inset; and (d) electric field in the cytoplasm as a function of the coordinate labeled $z$ for $x=0$ in the accompanying inset.

$\times 10^{-26} \mathrm{~J}$. The numerical energy that we will obtain in Sec. III for the analogous situation with the realistic model of the erythrocyte (i.e., the difference between the continuous line and the dashed line in Fig. 5(a) for $n=2$ ) is $W=-6.6$ $\times 10^{-27} \mathrm{~J}$. Since these calculations have been performed with the same volume of dielectric material (except for the small difference in the volume of the membrane), the difference is an estimate of the multipole and shape effects accounted for by our realistic calculations.

In order to calculate the modified transmembrane potential and the electric energy we have to determine the electric field distribution inside the uniform thickness membrane and the cytoplasm of the erythrocyte. As the cell dimensions $(\approx 8 \mu \mathrm{m})$ are much smaller than the wavelength at the working frequency $(\approx 2 \mathrm{~cm})$, we can assume that the cell is exposed to a uniform field. Therefore a quasistatic approximation holds and we can calculate the field distribution by solving directly the Laplace equation.

This approach neglects the double-layer effects associated to the presence and diffusion of counterions around the cell surface, which can modify significantly the membrane potential induced by external fields especially at low frequencies and when the conductivity of the external medium is low [40]. More quantitatively, this approximation will be justified whenever the two characteristic length scales of the system (the Debye screening length $\lambda_{D}$ that measures the extension of the counterion atmosphere and the distance $\Lambda$ an ion can diffuse during a field period) are smaller than the smallest length in the structure (the membrane thickness $\delta$ ). In a symmetric medium of permittivity $\epsilon$ and conductivity $\sigma$, $\lambda_{D}=\sqrt{\epsilon D / \sigma}$ and $\Lambda=\sqrt{4 D / \omega}$, where $D$ is the ion diffusion coefficient. At the physiological conditions and the frequency of $1.8 \mathrm{GHz}$ considered in this work and with a diffusion coefficient $D \approx 10^{-9} \mathrm{~m}^{2} / \mathrm{s}$ the ratios are $\lambda_{D} / \delta \approx 0.3$ and $\Lambda / \delta \approx 0.08$, thus validating our assumption.

Several researchers have used cell models based on shelled spheres or spheroids that permit analytical solutions for a variety of applications such as cell manipulation and EM field microdosimetry studies $[41,42]$. However, this analytical approach has severe limitations because an explicit solution of the Laplace equation usually requires a geometry consisting of one or several uniform media separated by interfaces which are constant-coordinate surfaces in certain types of suitable coordinate systems. This requirement excludes other possible geometric configurations, and even for spheroids the surface of the membrane has to be confocal with the main spheroid, often producing a nonuniform membrane thickness [31]. 
Therefore only numerical methods seem to be capable of giving a sufficiently precise estimation of field values in realistic cell shapes. But up to date very few studies of this type have been reported $[43,44]$, the main reason being the well-known difficulty in handling two regions-the cytoplasm and the membrane- of very different characteristic length scales. Since a numerical solution of the Laplace equation by a finite differences scheme ultimately involves some kind of polynomial approximation on the nodes of a convenient grid, the existence of a very small domain makes it necessary either to use a very dense grid (with a prohibitive cost in computing resources) or to resort to nonuniform meshing methods. Hybrid numerical techniques are a natural choice to overcome this difficulty [45], and very recently Liu et al. [46] have coupled the finite element method (FE) with the boundary element method (BEM) to compute the erythrocyte transmembrane potential in low frequency electric fields. However, they apply this coupling method only to an isolated erythrocyte cell.

In this paper we use a finite element technique [47,48] with a variable density mesh to determine the electric field intensity within the different layers of the cell. This method has been proven to be a powerful tool for the calculation of electric, magnetic and thermal fields. Although our adaptive mesh calculation exhibits a higher algorithmic complexity than the coupling method of Liu et al. [46], our technique can be readily applied to any number of neighboring erythrocytes within an arbitrarily shaped radiation region, making this approach perfectly suited for very accurate calculations of the electric fields in erythrocyte rouleau.

The cells are exposed to a linearly polarized EM plane wave of frequency $1.8 \mathrm{GHz}$ propagating along the $y$ axis, with the $\mathbf{E}$ and $\mathbf{H}$ field vectors parallel to the $z$ and $x$ axes. Two cases, the so-called electric polarization (where the electric vector $\mathbf{E}$ is aligned with the minor axis of the erythrocytes) and the so-called magnetic polarization (where the electric vector $\mathbf{E}$ is aligned with a major axis of the erythrocytes) are separately considered (cf. Fig. 1). The radiation region is taken to be a cube in which the cells are immersed and filled with the external medium and surrounded by perfectly matched layers (PML). This setup provides a reflectionless interface between the region of interest and the PML layers at all incident angles [49]. We achieve a good compromise between accuracy and computing resources by extending the dimensions of the radiation region to the order of four wavelengths in the external medium.

We set up an initial discretization of the object space into finite elements by a Delaunay algorithm [50]. This algorithm generates a mesh of tetrahedrons out of a set of node points and ensures the necessary topological compatibility as well as additional geometrical properties of the cell model. Once the initial mesh is established, a shape function is chosen inside each tetrahedron. With these shape functions we can obtain an initial solution of the full Maxwell equations. The values of the electric field at points inside each tetrahedron are interpolated from the values at the vertices of the tetrahedron, which must be sufficiently small to give sufficiently accurate initial values of the field inside. In order to reliably identify and selectively refine the large field intensity regions and large field-gradient regions (regions of high solution er- ror) that are developed within the cell structure, we have applied an adaptive finite element method (AFEM). There are different types of adaptive systems that have been developed for electromagnetic problems, and some are now in reasonably widespread use [51-53]. In this work, we have used a combined $h p$-type adaptive approach that independently varies the two basic discretization parameters, the sizes of elements within the mesh and the orders of elements in a mesh. This approach has the possibility and potential of realizing superior rates of solution error convergence compared against those methods that utilize only pure $h$-type or $p$-type adaption models [54-56]. The resulting (refined) matrix equation for the field values at the mesh nodes is solved iteratively. With this solution, we calculate the scattering parameters $S_{i j}$ of the reflected wave, and the process terminates when the difference between the values of $S_{11}$ and $S_{22}$ in two consecutive iterations is less than $10^{-6}$. We have found that the final mesh to reach a uniformly accurate result for the electric field $\mathbf{E}$ required 100000 tetrahedra per cubic micron of membrane and 1200 tetrahedra per cubic micron of cytoplasm. The calculation of the transmembrane potential is straightforward: we just multiply the electric field in the membrane by its thickness.

Similar FE calculations under the same EM field exposure are then set up for stacked copies of the original biconcave erythrocyte forming rouleau of up to ten cells. This FE numerical technique has already been validated by the authors in previous works with simpler spherical, cylindrical and spheroidal geometries $[9,23]$. A typical computing time for a rouleau of ten cells in a $3 \mathrm{GHz}$ microprocessor is of the order of $360 \mathrm{~min}$.

\section{MUTUAL INTERACTIONS AND ERYTHROCYTE ROULEAU ENERGY: ANALYSIS OF RESULTS}

Although extensive research has been carried out on the behavior of individual cells, much less effort has been devoted to the study of collective phenomena which may appear due to the electromagnetic properties of multiple cells subjected to an electromagnetic field. Mutual interactions between cells may lead to a significant change in the field distribution within each cell, as it is shown in the following analysis.

Figure 2 shows the interaction effects in the value of the transmembrane potential and electric field within the cytoplasm as a function of the distance $d$ between two erythrocyte neighbor cells. For cells stacked along the direction of the external electric field, the depolarizing fields lead to lower field intensity within the membrane and the cytoplasm as the distance $d$ decreases. Naturally, for higher values of $d$ the fields tend to the value for isolated erythrocytes. Note that the transmembrane potential values are smaller than those obtained when the erythrocyte is exposed to low frequency electric fields [46], because at high frequencies the dominant effect is the ratio of the permittivity of the external medium to the permittivity of the membrane, whereas at low frequencies the dominant effect is the ratio of the corresponding conductivities.

The variation of the electric field within a particular erythrocyte due to mutual interactions is much more significant as 

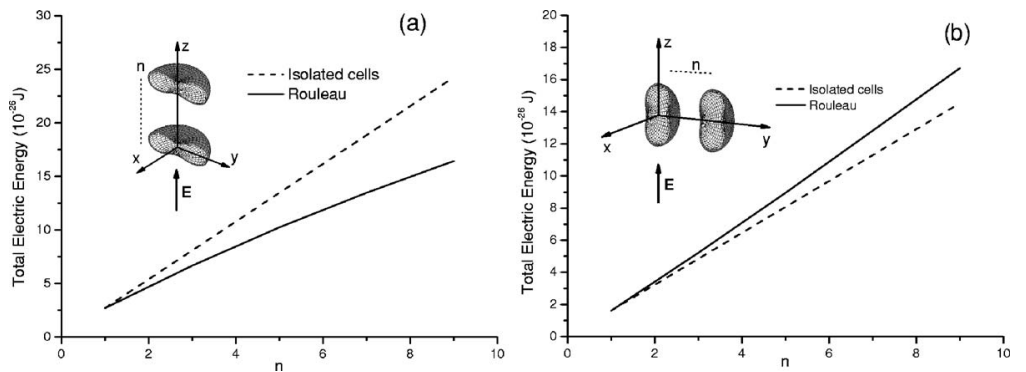

FIG. 5. Comparison between the total electric energy of an erythrocyte rouleau and the total energy of the same number of isolated erythrocytes in the same field of $1 \mathrm{~V} / \mathrm{m}$ when (a) the external field is parallel to the minor axes of the cells, and (b) the external field is parallel to the major axes of the cells. the number of intervening erythrocytes increases. To illustrate this effect, in Figs. 3(a) and 3(b) we plot the variation of the total field intensity along the major axis within the membrane and within the cytoplasm of a "central" cell as a rouleau is formed by stacking symmetrically new cells above and below the reference cell. The polarization of the external field is parallel to the minor axes of the cells, and separate curves correspond to the different values of $n$.

The corresponding results for the "magnetic" polarization of the field are plotted in Fig. 4. Since in this case there is no rotational symmetry around the field axis we present in separate plots the variation of the field in the membrane and in the cytoplasm along two perpendicular major axes of the cell: first the variation with the coordinate labeled $x$ in the accompanying insets and second the variation with the coordinate labeled $z$ in the insets. Note that for Fig. 4(a) the external field is approximately tangential to the membrane along a large part of the surface and therefore the field intensity exhibits very small variations with respect to the external field. The field within the membrane has much more significant variations in Fig. 4(c) but note that again when the external field is tangential to the membrane surface at $z \approx 3 \mu \mathrm{m}$ the electric field in the membrane equals the external field.

A comparison of Figs. 3 and 4 shows the influence of the polarization of the incident electromagnetic field: if the external field $\mathbf{E}$ is parallel to the major axis of the cell, the induced electric field in the membrane is lower than if the external field $\mathbf{E}$ is parallel to the minor axis of the cell. Similar results are obtained for the cytoplasm.

Note that although the membrane has been considered to be homogeneous in this latter case the field intensity at different points in the membrane varies very significantly, which in turn induces correspondingly large variations in the transmembrane potential. Thus, for the electric polarization the induced transmembrane voltage varies monotonically from a maximum of $70 \mathrm{nV}$ at the center to $8 \mathrm{nV}$ at the edge of the cell. However, when the field is magnetically polarized the maximum occurs at a point in the edge with minima at the center and at the points where the external field is tangential to the membrane surface. These results are in reasonable agreement with the induced transmembrane voltage of $50 \mathrm{nV}$ obtained by Foster and Schwan [57] with an spherical model of diameter equal to the diameter of the erythrocyte, and shows the role of a realistic model for the cell. We mention in passing that these induced values are very far away from the $100-300 \mathrm{mV}$ that would lead to the destruction of the membrane.

Our analysis also show the reduction of the field strength in the membrane near the regions of closest approach. This result is in agreement with the static calculations of Foster and Sowers [18] for two shelled spheres. Although Foster and Sowers point out that for their shelled spheres this effect is rather modest, in our realistic calculations the effect depends strongly on the polarization of the field and becomes more significant as the number of erythrocytes increases.

As we stated in Sec. I and in Sec. III, our main purpose is to evaluate the total electric energy involved in the formation of rouleau and to compare this energy with the total electric energy of isolated erythrocytes in the same external field. It turns out [58] that in the quasistatic approximation the electric energy of the rouleau embedded in the external medium can be written in terms of integrations extended only to the cell volume (membrane and cytoplasm)

$$
\begin{aligned}
W_{E}= & \frac{1}{2} \sum_{i=1}^{n}\left[\left(\epsilon_{\mathrm{ext}}-\epsilon_{\mathrm{cyt}}\right) \int_{V_{\mathrm{cyt}}} \mathbf{E}_{i, \mathrm{cyt}} \cdot \mathbf{E}_{\mathrm{ext}} d V\right. \\
& \left.+\left(\epsilon_{\mathrm{ext}}-\epsilon_{\mathrm{mem}}\right) \int_{V_{\mathrm{mem}}} \mathbf{E}_{i, \mathrm{mem}} \cdot \mathbf{E}_{\mathrm{ext}} d V\right],
\end{aligned}
$$

where the subindexes "cyt" and "mem" stand for cytoplasm and membrane, respectively, and where $n$ is the number of cells in the rouleau. Note again the role of the polarization of the external field in Eq. (7): only the component of the induced fields in the direction of the external field enter in the calculation of the energy. We have evaluated the integrals in Eq. (7) numerically using the field values for the cytoplasm and the membrane discussed in the previous paragraphs.

Figure 5(a) shows the total electric energy for the rouleau as a function of the number of rouleau cells. The applied external field has "electric" polarization (external field parallel to the minor axis of the cell). A significant reduction of the total electric energy of the stack is found as the rouleau gets longer. This reduction is a consequence of the decrease in the induced fields already discussed. In Fig. 5(a) the rouleau energy is also compared with the energy of the same number of isolated erythrocytes (the dashed straight line). Therefore, we conclude that an external field "electrically" polarized favors the rouleau formation, because the total energy system decreases.

However, this conclusion does not hold for the case where the external field has "magnetic" polarization (that is, the electric field is parallel to the cell major axis). Figure 5(b) shows the total electric energy of the rouleau for this polarization of the external field. Indeed, by comparing Figs. 5(a) and 5(b) we find two significant differences. First, for the same number of cells, the total electric energy of the rouleau formed under exposure of a polarized external field parallel 
to the minor axis has a significant lower value compared to the rouleau formed under a polarized external field parallel to the major axis, due to the lower electric fields induced in the membrane and cytoplasm of each erythrocyte as the number of rouleau cells increases (Fig. 3). The second and most important difference is that the total rouleau energy is higher than the energy of a group of identical number of isolated erythrocytes exposed to the same external field. Therefore, it can be concluded that an external field that is polarized parallel to the major axis could favor the disaggregation of the rouleau, because the total system energy would increase.

The calculated energy differences for both orientations of the electric field as shown in Figs. 5(a) and 5(b) are in qualitative agreement with an interpretation in terms of the induced dipoles in the erythrocytes that form the rouleau. The interaction energy between two electric dipoles with (complex) dipole moments $\mathbf{p}_{1}$ at $\mathbf{r}_{1}$ and $\mathbf{p}_{2}$ at $\mathbf{r}_{2}$ with arbitrary orientations is

$$
W=\frac{\operatorname{Re}\left(\mathbf{p}_{1} \mathbf{p}_{2}^{*}\right)}{8 \pi \epsilon_{\mathrm{ext}} r^{3}}\left(\cos \alpha-3 \cos \theta_{1} \cos \theta_{2}\right),
$$

where $\alpha$ is the angle between the (real) moments and $\theta_{1}$ and $\theta_{2}$ the angles that they form with $\mathbf{r}=\mathbf{r}_{2}-\mathbf{r}_{1}$. If the external field is applied along the minor axis of the cell, the induced dipoles are aligned parallel to the rouleau axis and therefore their interaction energy is negative. Conversely, if the applied field is parallel to the major axis of the erythrocytes, the induced dipoles are arranged perpendicularly to the rouleau direction, thus giving a positive contribution to the total energy of the rouleau with respect to the energy of free erythrocytes.

\section{CONCLUSIONS}

In the present paper we have calculated numerically the effect of an electromagnetic field on the electric field distribution in the membrane and cytoplasm of erythrocytes. These cells have been modeled as two-compartment dielectric particles with a realistic biconcave shapes obtained by an appropriate parametrization based on the dimensions of human erythrocytes. Our numerical approach has been a FE technique, with adaptive nonuniform meshing to take into account the small thickness of the membrane, producing a uniformly accurate computation of internal fields. Using this method we have studied the influence of neighboring cells on the electric field distribution in a given erythrocyte during rouleau formation.

Although our analysis has some simplifying assumptions (essentially a rouleau composed of identical nondeformed erythrocytes and a negligible influence of counterion double layer), the results clearly show an influence of the number of stacked cells on the value of the electric field in the membrane and in the cytoplasm of the central cell of the rouleau. This influence has a different sign for the cases of orientation of the electric field along the minor and along the major axes of the erythrocyte. The general pattern can be understood as a result of the interaction between the induced dipoles in neighboring cells polarized by the electric field, although we have shown that quantitative results require careful numerical calculations.

The comparison of the energies of free erythrocytes and aggregated erythrocytes revealed that the rouleau formation is energetically favorable when the field orientation is along the minor axis of the erythrocytes and unfavorable when the orientation is parallel to their major axis, i.e., as we anticipated in Sec. I field-induced aggregation is favored along the field lines. For the size of the erythrocyte considered in this analysis, the energy differences found for an external field of $1 \mathrm{~V} / \mathrm{m}$ are much smaller than the adhesion energy between normal aggregated erythrocytes [2]. However, it has to be taken into account that the energy is proportional to the square of the field intensity and therefore higher fields can affect erythrocyte aggregation. We also recall that although in our analysis the membrane has been considered as an homogeneous dielectric shell, the electric field in the membrane and the ensuing transmembrane potential varies significantly. However, the cell membrane is a very complex structure and inhomogeneities such as proteins or ionic channels can enhance the electric field, leading to higher field spots and therefore modifying the aggregation forces. Taking into account these dynamical effects in a realistic calculation is a challenging problem.

The model developed herein gives insight into electrical influences between cells immersed in an electromagnetic field which have not been previously addressed because of the limitations of analytical approaches and the difficulties of accurate numerical computations. Application of this model and refinements thereof may be of potential value in understanding possible hazards or beneficial uses of electromagnetic field radiation.

\section{ACKNOWLEDGMENTS}

This work was supported by the Fondo de Investigaciones Sanitarias under Grant No. PI03/0295 and by the Spanish Ministerio de Ciencia y Tecnología under Project No. BFM2002-02646.
[1] R. Ben-Ami, G. Barshtein, T. Mardi, V. Deutch, O. Elkayam, S. Yedgar, and S. Berliner, Am. J. Physiol. 285, H2663 (2003).

[2] R. Skalak, P. Zarda, K. M. Jan, and S. Chien, Biophys. J. 35, 771 (1981).

[3] B. Bozic, S. Svetina, and B. Zeks, Phys. Rev. E 55, 5834
(1997)

[4] J. Derganc, B. Bozic, S. Svetina, and B. Zeks, Biophys. J. 84, 1486 (2003).

[5] Z. Qin, L. G. Durand, L. Allard, and G. Cloutier, Med. Biol. 24, 503 (1998).

[6] J. Gimsa and D. Wachner, Eur. Biophys. J. 30, 463 (2001). 
[7] J. C. Lin, A. W. Guy, and C. C. Johnson, IEEE Trans. Microwave Theory Tech. 23, 246 (1975).

[8] J. L. Sebastián, S. Muñoz San Martín, M. Sancho, and J. M. Miranda, Phys. Med. Biol. 46, 213 (2001).

[9] S. Muñoz San Martín, J. L. Sebastián, M. Sancho, and J. M. Miranda, Phys. Med. Biol. 48, 1649 (2003).

[10] M. Saito and H. P. Schwan, Biological Effects of Microwave Radiation (Plenum, New York, 1961).

[11] H. P. Schwan and L. D. Sher, J. Electrochem. Soc. 116, 22C (1969).

[12] L. D. Sher, E. Kresh, and H. P. Schwan, Biophys. J. 10, 970 (1970).

[13] S. Takashima and H. P. Schwan, Biophys. J. 47, 513 (1985).

[14] F. A. Sauer, in Coherent Excitations in Biological Systems, edited by H. Frölich K. Kremer (Springer, New York, 1983), pp. 134-144.

[15] L. D. Sher, Ph.D. thesis, University of Pennsilvania, 1969.

[16] H. P. Schwan, Br. J. Cancer 45, 513 (1982).

[17] F. A. Sauer, in Interactions between Electromagnetic Fields and Cells, edited by A. Chiabrera, C. Nicolini, and H. P. Schwan (Plenum, New York, 1985), pp. 181-202.

[18] K. R. Foster and A. E. Sowers, Biophys. J. 69, 777 (1995).

[19] J. Thuéry, Microwaves: Industrial, Scientific and Medical Applications (Artech House, Norwood, 1992).

[20] O. P. Gandhi and G. Kang, Phys. Med. Biol. 47, 1501 (2002).

[21] J. B. Van de Kamer and J. W. Lagendijk, Phys. Med. Biol. 47, 1827 (2002).

[22] M. Martínez-Burdalo, A. Martín, M. Anguiano, and R. Villar, Phys. Med. Biol. 49, 345 (2004).

[23] J. L. Sebastián, S. Muñoz San Martín, M. Sancho, and J. M. Miranda, Bioelectrochemistry 64, 39 (2004).

[24] A. Gray, Modern Differential Geometry of Curves and Surfaces with Mathematica (CRC Press, Boca Raton, 1998).

[25] P. Moon and D. E. Spencer, Field Theory Handbook: Including Coordinate Systems, Differential Equations and Their Solutions (Springer, Berlin, 1998).

[26] M. I. G. Bloor and M. J. Wilson, Phys. Rev. E 61, 4218 (2000)

[27] Y. Jie, L. Quanhui, L. Jixing, and O. -Y. Zhong-Can, Phys. Rev. E 58, 4730 (1998).

[28] P. Kuchel and E. Fackerell, Bull. Math. Biol. 61, 209 (1999).

[29] S. Wolfram, The Mathematica Book (Wolfram Media, Champaign, 2003).

[30] L. Stryer, Biochemistry (Freeman, New York, 1995).

[31] J. Gimsa and D. Wachner, Biophys. J. 81, 1888 (2001).

[32] J. P. Huang and K. W. Yu, Commun. Theor. Phys. 39, 506 (2003).

[33] R. Martinsen, S. Grimnes, and H. Schwan, Encyclopedia of
Surface and Colloid Science (Marcel Dekker, New York, 2002), pp. 2643-2652.

[34] J. Z. Bao, C. C. Davis, and R. E. Schmukler, Biophys. J. 61, 1427 (1992).

[35] M. Simeonova, D. Wachner, and J. Gimsa, Bioelectrochemistry 56, 215 (2002).

[36] L. Miao, U. Seifert, M. Wortis, and H. G. Döbereiner, Phys. Rev. E 49, 5389 (1994).

[37] U. Seifert and R. Lipowsky, Phys. Rev. A 42, 4768 (1990).

[38] H. Darmani and W. T. Coakley, Biochim. Biophys. Acta 1021, 182 (1990).

[39] T. B. Jones, Electromechanics of Particles (Cambridge University Press, Cambridge, 1995).

[40] C. Grosse and H. Schwan, Biophys. J. 63, 1632 (1992).

[41] L. M. Liu and S. F. Cleary, Bioelectromagnetics (N.Y.) 16, 160 (1995).

[42] P. Bernardi, M. Cavagnaro, X. d'Inzeo, and M. Liberti, URSI XXVI General Assembly, 1999, p. 616.

[43] C. E. Miller and C. S. Henriquez, IEEE Trans. Biomed. Eng. 35, 712 (1988).

[44] S. Muñoz San Martín, J. L. Sebastián, M. Sancho, and J. M. Miranda, Bioelectromagnetics (N.Y.) 25, 631 (2004).

[45] G. C. Hsiao, Z. Angew. Math. Mech. 70, 493 (1990).

[46] C. Liu, D. Sheen, and K. Huang, IEEE Trans. Nanobiosci. 2, 104 (2003).

[47] J. Jin, The Finite Element Method in Electromagnetics (Wiley, New York, 1993).

[48] J. Douglas, Jr., J. E. Santos, and D. Sheen, Math. Models Meth. Appl. Sci. 10, 593 (2000).

[49] E. Becache and P. Joly, Tech. Rep. 4164, INRIA, 2001.

[50] D. N. Shenton and Z. J. Cendes, IEEE Trans. Magn. 21, 2535 (1985)

[51] S. McFee and J. P. Webb, IEEE Trans. Magn. 21, 2535 (1992).

[52] S. McFee and D. Giannacopoulos, IEEE Trans. Magn. 32, 1357 (1996).

[53] P. Fernandes, P. Girdinio, M. Repetto, and G. Secondo, IEEE Trans. Magn. 28, 1739 (1992).

[54] D. Giannacopoulos and S. McFee, IEEE Trans. Magn. 30, 3523 (1994).

[55] S. McFee and D. Giannacopoulos, IEEE Trans. Magn. 34, 3284 (1998).

[56] W. Gui and I. Babuska, Numer. Math. 49, 613 (1986).

[57] K. R. Foster and P. H. Schwan, in Handbook of Biological Effects of Electromagnetic Radiation, edited by C. Polk and E. Postow (CRC, Boca Raton, 1995), pp. 25-102.

[58] J. A. Stratton, Electromagnetic Theory (McGraw-Hill, New York, 1941). 